\section{María Alejandra Belaúnde \\ Gómez' \\ Fernando Salazar Silva ${ }^{2}$ \\ Diana Castillo-Andamayo ${ }^{2}$ Jorge Manrique Chávez ${ }^{2}$ Francisco Orejuela Ramírez ${ }^{2}$ Carol Zavaleta Boza ${ }^{2}$ Martha López Pinedo ${ }^{3}$}

'Bachiller en Estomatologia. Universidad Peruana Cayetano Heredia.

2Doctor en Estomatología. Docente del Departamento Académico de Odontología Social. Facultad de Estomatología Roberto Beltrán. Universidad Peruana Cayetano Heredia. Lima, Perú.

${ }^{3}$ Magister en Estomatología. Docente del Departamento Académico de Clínica del Adulto. Facultad de Estomatología Roberto Beltrán. Universidad Peruana Cayetano Heredia. Lima, Perú.

\section{Correspondencia}

María Alejandra Belaúnde Gómez Calle María Parado de Bellido 157 Lima, Perú

E-mail: maria.belaunde@upch.pe

\title{
Asociación del acceso a la atención dental y el edentulismo.
}

Belaunde-Gomez A, Salazar-Silva F ${ }^{1}$, Castillo-Andamayo D², Manrique-Chávez J², Orejuela-Ramírez $\mathrm{F}^{2}$, Zavaleta-Boza $\mathrm{C}^{2}$, López-Pinedo $\mathrm{ML}^{3}$. Asociación del acceso a la atención dental y el edentulismo. Rev Estomatol Herediana. 2012; 22(2):77-81

\section{RESUMEN}

El acceso a la atención se basa en la capacidad del individuo para obtener atención dental cuando sea necesario, o ante algunas barreras que podrían impedir la atención. Objetivo: Determinar la relación entre el acceso a la atención dental y el edentulismo. Material y métodos: La investigación fue transversal, observacional y descriptiva. La muestra fue de 148 personas adultas entre 18 a 65 años de edad del distrito de Cajabamba, provincia de Cajabamba, departamento de Cajamarca. El acceso a la atención dental se evaluó mediante tres variables que son: razón por la cual la última vez no pudo obtener cuidado dental, razón principal de última visita al dentista y última visita al dentista. Se realizó un análisis univariado para evaluar la distribución de cada una de las variables, de tal manera que se pudo detectar valores fuera de lo previsto. También se efectuó un análisis bivariado, mediante la prueba de Chi cuadrado, para determinar la relación entre las variables componentes de acceso a la atención dental y el edentulismo. Las pruebas se realizaron con el programa Stata Inter Cooled 8.0. Resultados: El 100\% de edéntulos totales superiores y parciales inferiores acudieron al dentista por última vez por presentar dolor, mientras que el 100\% de edéntulos totales acudió para la confección de prótesis. En mayor proporción los dentados visitaron por última vez al dentista en un tiempo menor a 12 meses siendo el 33,73\%, y con un 36,36\% los edéntulos totales y edéntulos parcial bimaxilar acudieron en mayor proporción en un tiempo mayor a 5 años. Conclusión: Existe relación entre el acceso a la atención dental y el edentulismo.

Palabras Clave: EDENTULISMO, ACCESO, ATENCIÓN DENTAL.

\section{Association between Access to dental care and Edentulism}

\section{ABSTRACT}

Access to care refers to propensity of the individual to obtain any services, and the capacity of the system to match the patient's needs and preferences with appropriate level of services. Objective: Determine the association between access to dental care and edentulism. Material and methods: This was an observational descriptive cross-sectional study. The sample included 18-65 years old adults from the district of Cajabamba, Cajamarca, Peru. Access to dental care was measured using questions. The distribution of each variable was explored in univariate analysis. The association between access to dental care and edentulism were tested using the chi-square test. The outcome of the research was obtained using Stata Inter Cooled 8.0. Results: All edentulous maxilla and partially edentulous jaw ??? sought the dentist for the last time due to pain, meanwhile $100 \%$ of edentulous patients received dental prosthetic treatments. The majority of the patients visited the dentist one last time in a period less than 12 months being the $33.73 \%$, and $36.36 \%$ total edentulous y partial bimaxilar sought dental care in a larger proportion in a 5 year time period. Conclusion: There exists a relationship between the access to dental care and edentulism.

Key Words: EDENTULISM, ACCESS, DENTAL CARE.

\section{Introducción}

A nivel mundial, en los adultos se ha encontrado una deficiente higiene oral evidenciándose así un alto nivel de pérdida de piezas dentarias, caries dental, alta prevalencia de enfermedad periodontal, xerostomía y lesiones premalignas o cáncer oral (1). Siendo más prevalente la pérdida de piezas dentarias, el impacto es más negativo en personas edéntulas ya que la pérdida de dientes reduce el rendimiento de la función masticatoria afectando en la elección de los alimentos, consecuentemente causando la pérdida de peso en las personas afectadas. El edentulismo además de causar problemas en la masticación, trae desventajas sociales como en la comunicación1. Es más prevalente en los adultos mayores en todo el mundo y está asociado al nivel socio-económico. En varios estudios epidemiológicos muestran que los adultos mayores con nivel socioeconómico bajo con o sin nivel educativo presentan más índices de edentulismo que las personas adultas mayores con mejor nivel socioeconómico y con educación (1).

El edentulismo afecta la salud oral, la salud en general y la calidad de vida de las personas afectadas ya que varía la dieta alimenticia, pue- 
de variar el gusto de los alimentos trayendo como consecuencia la malnutrición y desnutrición. Aún las personas que usan prótesis ya sean parciales o totales no tienen la misma efectividad masticatoria que teniendo todas las piezas dentarias. Siendo los principales contribuyentes al edentulismo la enfermedad periodontal crónica y la caries dental (2). Aunque en algunos estudios indican que la caries dental es la razón más importante para realizar la extracción dental en comparación con la enfermedad periodontal (3).

La pérdida de dientes afecta a las personas de diferentes edades, pero se observa más en los adultos ya que por el tiempo de vida han desarrollado enfermedades crónicas como la caries dental y la periodontitis. Sin embargo no es un resultado inevitable del envejecimiento. Por otra parte el impacto de la promoción de la salud oral y las acciones públicas como el consumo de agua fluorada, sugieren que el edentulismo se puede prevenir (4).

El acceso a la atención es la capacidad de un individuo para obtener servicios y la capacidad del sistema para satisfacer las necesidades y las preferencias de los pacientes con un nivel adecuado de servicios (5), es así, que el acceso a la atención dental se basa en la capacidad del individuo para obtener atención dental cuando sea necesario, o ante barreras que podrían impedir la atención del individuo.

El seguimiento al acceso y a la utilización de servicios de un grupo, se considera importante, ya que se basa en la suposición de que el nivel de uso de servicios dentales está directamente relacionado con el estado de la salud bucal; entonces, los individuos que usen los servicios de atención dental deberían estar más saludables en comparación con aquellos que no lo hacen (6).

Para conseguir la atención, una persona debe tener por lo menos dos cosas: la disponibilidad de un consultorio dental privado o el consultorio en un hospital o puesto de salud, y un medio para pagar la atención que necesita, la falta de tales medios será la barrera más común para acceder a la atención dental, siendo las familias de bajos ingresos las más perjudicadas (7).

\section{Material y Métodos}

La presente investigación fue de tipo descriptivo, observacional de corte transversal. La muestra estuvo conformada por 148 personas adultas entre los 18 a 65 años de edad del distrito de Cajabamba, provincia de Cajabamba, departamento de Cajamarca. La muestra se seleccionó empleando el muestreo no aleatorio, por conveniencia. Participaron en el presente estudio, los pacientes que firmaron un consentimiento informado, el cual fue aprobado por el Comité Institucional de Ética de la Universidad Peruana Cayetano Heredia.

La participación fue voluntaria y las personas que aceptaron ser parte de la investigación completaron un cuestionario, el cual estaba conformado por preguntas que respondían si los pobladores tenían el acceso a la atención dental, también se evaluó clínicamente a los participantes para clasificarlos en: dentado completo, edéntulo parcial superior, edéntulo parcial inferior, edéntulo parcial bimaxilar, edéntulo total superior y parcial inferior, y edéntulo total.
El acceso a la atención dental fue medido de acuerdo a las variables: razón por la cual la última vez no pudo obtener cuidado dental, razón principal de última visita al dentista y última visita al dentista. Se realizó el análisis univariado para evaluar la distribución de cada una de las variables, de tal manera que se pudo detectar valores fuera de lo previsto; y también se efectuó el análisis bivariado, mediante la prueba de Chi cuadrado, para determinar la relación entre las variables componentes de acceso a los servicios de atención dental y el edentulismo. Las pruebas se realizaron con el programa Stata Inter Cooled 8.0.

\section{Resultados}

Se encuestaron a 148 adultos de 18 a 65 años de edad. La tabla 1 muestra que el $45,24 \%$ de dentados completos y el $36,84 \%$ de la condición parcial superior no acudieron al dentista por falta de dinero. El $50 \%$ de la condición parcial inferior, el $41,18 \%$ de la condición parcial bimaxilar y el $50 \%$ de la condición total superior y parcial inferior, no acudieron la última vez al dentista por falta de tiempo; mientras que el $100 \%$ de edéntulos totales y el $50 \%$ de la condición total superior y parcial inferior no fueron al dentista por otras razones. Al comparar las variables de condición de dentición y razón por la cual no fue al dentista se obtuvieron resultados los cuales son estadísticamente significativos.

En la tabla 2, se evidencia que el $100 \%$ de edéntulos totales superiores y parciales inferiores acudieron al dentista por última vez por presentar dolor, mientras que el $100 \%$ de edéntulos totales acudió para la confección de prótesis. 
Tanto los dentados completos como los edéntulos parciales superiores y los edéntulos parciales bimaxilares acudieron por última vez al dentista para que les realizaran una limpieza dental, representando el 57,14\%, $31,58 \%$ y el $29,41 \%$ respectivamente. El 37,5\% de los edéntulos parciales inferiores recibieron como tratamiento las curaciones dentales.

La tabla 3 muestra que la mayor proporción en dentados que visitó por última vez al dentista fue el
$33,73 \%$, quienes acudieron al dentista en un tiempo menor a 12 meses; y el valor menor se encontró en la última visita en un tiempo mayor a 5 años con un 9,09\%. Cabe resaltar que solo hubo tres casos que nunca recibieron atención dental, los cuales son dentados y representan un total del $100 \%$. Los edéntulos parciales bimaxilares son los que obtuvieron los porcentajes más elevados. El $62,5 \%$ visitó por última vez al dentista en el tiempo de 1 a 2 años, el 44,58\% visitaron por última vez al dentista en un tiempo menor a 12 meses y el $36,36 \%$ visitó al dentista en un tiempo mayor a 5 años. El otro grupo que tuvo las proporciones elevados son los edéntulos totales; el 36,36\% visitó por última vez al dentista en un tiempo mayor a 5 años, el 27,27\% lo visitó entre 3 a 5 años. Mientras que la menor proporción de todos los edéntulos la obtuvieron los edéntulos totales con un $5 \%$ en el tiempo de 1 a 2 años. Al relacionar las variables de este estudio se en-

Tabla 1. Relación de condición de dentición y razón por la cual la última vez no pudo obtener cuidado dental

\begin{tabular}{lcccccc}
\hline \multirow{2}{*}{ Condición de dentición } & \multicolumn{3}{c}{ Razón por la cual la última vez no pudo obtener cuidado dental } \\
\cline { 2 - 7 } & \multicolumn{2}{c}{ Tiempo } & \multicolumn{3}{c}{ Dinero } & \multicolumn{3}{c}{ Otros } \\
\cline { 2 - 7 } & $\mathrm{n}$ & $\%$ & $\mathrm{n}$ & $\%$ & $\mathrm{n}$ & $\%$ \\
\hline Dentado completo & 15 & 35,71 & 8 & 19,05 & 19 & 45,24 \\
E. Parcial superior & 6 & 31,58 & 6 & 31,58 & 7 & 36,84 \\
E. Parcial inferior & 4 & 50 & 2 & 25 & 2 & 25 \\
Parcial & 28 & 41,18 & 20 & 29,41 & 20 & 29,41 \\
E. Total superior y parcial inferior & 1 & 50 & 0 & 0 & 1 & 50 \\
Edéntulo & 0 & 0 & 0 & 0 & 9 & 100 \\
\hline
\end{tabular}

$\mathrm{p}=0,033$

Tabla 2. Relación de condición de dentición y razón principal de última visita al dentista.

\begin{tabular}{|c|c|c|c|c|c|c|c|c|c|c|c|c|}
\hline \multirow{3}{*}{ Condición de dentición } & \multicolumn{12}{|c|}{ Razón principal de última visita al dentista } \\
\hline & \multicolumn{2}{|c|}{ Curaciones } & \multicolumn{2}{|c|}{$\begin{array}{c}\text { Para tener } \\
\text { prótesis }\end{array}$} & \multicolumn{2}{|c|}{$\begin{array}{c}\text { No ha ido al } \\
\text { dentista }\end{array}$} & \multicolumn{2}{|c|}{ Extracciones } & \multicolumn{2}{|c|}{ Profilaxis } & \multicolumn{2}{|c|}{ Dolor } \\
\hline & $\mathrm{n}$ & $\%$ & $\mathrm{n}$ & $\%$ & $\mathrm{n}$ & $\%$ & $\mathrm{n}$ & $\%$ & $\mathrm{n}$ & $\%$ & $\mathrm{n}$ & $\%$ \\
\hline Dentado completo & 8 & 19,05 & 0 & 0 & 3 & 9,52 & 1 & 2,38 & 25 & 57,14 & 5 & 11.9 \\
\hline E. Parcial superior & 3 & 15,79 & 1 & 5,26 & 0 & 0 & 5 & 26,32 & 6 & 31,58 & 4 & 21.05 \\
\hline E. Parcial inferior & 3 & 37,5 & 1 & 12,5 & 0 & 0 & 0 & 0 & 2 & 25 & 2 & 25 \\
\hline Parcial & 8 & 11,76 & 12 & 17,65 & 0 & 0 & 14 & 20,59 & 20 & 29,41 & 14 & 20.59 \\
\hline E. Total superior y parcial inferior & 0 & 0 & 0 & 0 & 0 & 0 & 0 & 0 & 0 & 0 & 2 & 100 \\
\hline Edéntulo & 0 & 0 & 9 & 100 & 0 & 0 & 0 & 0 & 0 & 0 & 0 & 0 \\
\hline
\end{tabular}

Tabla 3. Relación de última visita al dentista según condición de dentición

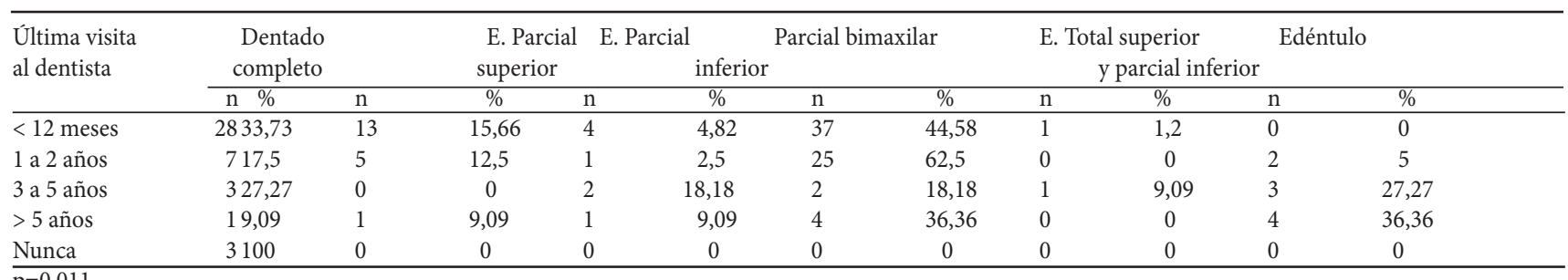

$\mathrm{p}=0,011$ 
tró el valor de $\mathrm{p}=0,011$ siendo los resultados estadísticamente significativos.

\section{Discusión}

En el Perú, el 84\% de los niños presentan caries dental a los 12 años de edad con un promedio de 3.1 dientes permanentes con experiencia de caries dental. En países en vías de desarrollo se observa este alto índice de experiencia de caries dental, y se debe a que en estos países no se han establecido normas para que se realicen actividades comunitarias de tipo preventivo-promocional (8).

En la bibliografía actual no se encontró ninguna investigación que analice la relación entre el acceso a la atención dental y el edentulismo en adultos jóvenes, sin embargo si existen trabajos de investigación en adultos mayores.

Abrams y col. encontraron que en el grupo etario, mayores a 55 años con el 38,5\% citaron temor y falta de dinero como razones por las cuales no acudieron al dentista, mientras que casi el doble fue citado como "no necesita" siendo el 72,5\% $(9,10)$. Mientras que Kiyak encontró que el $90 \%$ de adultos mayores dieron como respuesta que no necesitaban ir al dentista $(9,11)$. Estos estudios coinciden con la presente investigación, ya que el $100 \%$ de edéntulos totales (quienes son mayores de 65 años) (Tabla 1) dieron como razón por la cual no van al dentista "otros motivos" $y$ en esos otros motivos se encuentra la falta de percepción de necesidad de cuidado dental. También el 50\% de la condición de edentulismo total superior y parcial inferior dieron la misma respuesta.
En un estudio de Australia se encontró que en el grupo etario de 60 a 74 años de edad acudió al dentista en mayor proporción por curaciones, seguidamente por las extracciones dentales y por último en menor proporción, acudieron para la confección de prótesis (12). La población estudiada acudió mayormente al dentista para una limpieza dental, ya sean edéntulos o dentados completos; existe cierta discrepancia ya que la mayor proporción son edéntulos, entonces la razón principal de última visita al dentista debería de ser la extracción dental; sin embargo, gracias a la existencia de programas preventivos-promocionales por parte del Hospital de Apoyo Cajabamba y también gracias al trabajo preventivo de los internos de estomatología de la UPCH se ha obtenido este resultado.

Mann y col evaluaron a 87 personas y encontró una diferencia estadísticamente significativa en la última visita al dentista en relación a los dentados y a los edéntulos, siendo el $p<0,0063$ (13). Dicho estudio coincide con los resultados obtenidos en la presente investigación, al comparar la última visita al dentista en relación a la condición de dentición se encontró el valor $p=0,011$ (Tabla 3). Está comparando el estudio de la referencia 13 con la tabla 3 del presente estudio. A pesar del valor $\mathrm{p}$, los resultados de la presente investigación solo coinciden en el caso de los edéntulos ya que fueron los que en mayor proporción visitaron por última vez al dentista en un tiempo mayor a 5 años; mientras que Mann y col encontraron que el $61,1 \%$ los dentados fueron al dentista en un tiempo menor a 12 meses y los edéntulos $38,9 \%$ lo cual es totalmente opues- to en nuestro estudio ya que el $33,73 \%$ de dentados y el $66,27 \%$ de edéntulos acudieron al dentista en un tiempo menor a 12 meses.

Algunos de los resultados de la investigación no coinciden con la literatura comparada, y esto se debe a que la muestra del presente estudio se tomó por conveniencia; también se debe a que la población estudiada es joven ya que toma un rango de edades de 18 a 65 años de edad; mientras que los estudios en diversos artículos mencionados toman como muestra a poblaciones adultas mayores, de 55 años en adelante.

Por ello, es momento de implementar nuevas metodologías en los programas de salud los cuales permitan el mejoramiento del acceso a la atención dental, logrando educar a largo plazo a la población y así evitar la pérdida de piezas dentarias en adultos jóvenes.

Una de las limitaciones del estudio fue que la investigación fue de tipo descriptivo, de tal manera que la información no se pudo generalizar. Sin embargo el presente estudio servirá como una guía para las próximas investigaciones ya que no hay información de la realidad del Perú sobre este tema ya que no se encontrò literatura sobre estudios de este tipo a a poblaciones adultas jóvenes.

\section{Referencias Bibliográficas}

1. Petersen PE, Yamamoto T. Improving the oral health of older people: the approach of the World Health Organization Global Oral Health Programme. Community Dent Oral Epidemiol. 2005;33:81-92.

2. Petersen PE, Bourgeois D, Ogawa H, Estupinan-Day S, 
Ndiaye C. The global burden of oral diseases and risks to oral health. Bull World Health Org. 2005;83:661-9.

3. Muller F, Naharro M, Carlsson GE. What are the prevalence and incidence of tooth loss in the adult and elderly population in Europe?. Clin Oral Impl Res. 2007;18(3):2-14

4. Cooper LF. The current and future treatment of edentulism. J Prosthodont. 2009;18:116-122.

5. Petersen PE. The World Oral Health Report 2003: continuous improvement of oral health in the 21 st century - the approach of WHO Global Oral Health Programme. Com Dent Oral Epid. 2003;31(1):3-24.

6. Dolan TA, Atchison KA. Implications of access, utilization and need for oral health care by the non-institutionalized and institutionalized elderly on the dental delivery system. J Dent Educ. 1993;57(12): 876-87.
7. Ohio Department of Health. Oral health and access to dental care for Ohioans. Ohio, USA: Ohio Department of Health; 2010.

8. Bernabé OE, Sánchez BP, Delgado AE. Efectividad de una intervención comunitaria en salud oral: resultados después de 18 meses. Rev Med Hered. 2006;17(3):170-76.

9. Kiyak HA, Reichmuth M. Barriers to and enablers of older aldult's use of dental services. J Dent Educ. 2005; 69(9): 975 86.

10.Abrams RA, Ayers CS, Lloyd PM. Attitudes of older versus younger adults toward dentistry and dentists. Spec Care Dent. 1992:12:67-70.

11.Kiyak HA. An explanatory model of older persons' use of dental services: implications for health policy. Med Care. 1987;25:936-52.

12.Dental Statistics and Research
Unit. Oral health and access to Dental Care - older adults in Australia. Canberra: Australian Institute of Health \& Welfare; 2000.

13. Mann J, Mersel A, Ernest M, Labiv M. Dental behavioral aspects of a non-institutionalized elderly population. Gerodontology. 1990;9(3):83-7. 